\title{
Breakthrough: Ibuprofen/nitazoxanide/azithromycin: a battle changer personalized COVID-19 telemedicine five days protocol
}

\author{
Mina Kelleni ${ }^{1}$ \\ ${ }^{1}$ Minia University
}

June 22, 2020

\begin{abstract}
Until recently, ibuprofen has been avoided in all COVID-19 protocols world-wide. The author has been trying since March, 2020 to publish a paper that disputes this unfortunate incident and he finally managed since May 2020 to publish two manuscripts that not only prove non-steroidal anti-inflammatory drugs not harmful for COVID-19, rather they were shown to possess a potential to cure the disease based on a unique new theory suggested to explain COVID-19 pathogenesis and he suggested NSAIDs to be added to unique protocol he suggested using nitazoxanide/azithromycin to manage early cases of COVID19. In this manuscript, considered the fourth related to the subject, the author represents the first clinical results of using NSAIDs/Nitazoxanide/Azithromycin protocol, used partly or fully, that includes relatively cheap FDA approved drugs used in seventeen Egyptian patients, whether confirmed or suspected, including children, adults and two pregnant ladies whom have been mostly symptoms-free in five days. The manuscripts also presents a road-map to illustrate how to deal efficiently with early cases of COVID-19 according to the author's clinical experience.
\end{abstract}

Running head: Ibuprofen/Nitazoxanide/Azithromycin for COVID-19

Title: Breakthrough: Ibuprofen/nitazoxanide/azithromycin: a battle changer personalized COVID-19 telemedicine five days protocol

Author and Principal investigator

Mina T. Kelleni, MD, PhD

Assistant Professor of Pharmacology, College of Medicine, Minia University, Egypt.

Assistant Professor of Pharmacology, College of Pharmacy, Jouf University, KSA.

Mobile: +966560407874

drthabetpharm@yahoo.com, mina.kelleni@mu.edu.eg, mtkelleni@ju.edu.sa

https://orcid.org/0000-0001-6290-6025

Two sentenced Biography

Physician, assistant Professor of Pharmacology, member of the editorial boards of numerous international medical journals related to internal medicine, diabetes and obesity among other specialties.

WHAT IS ALREADY KNOWN ABOUT THIS SUBJECT

It's known that paracetamol is the drug of choice to manage COVID-19 associated fever

It's known that Ibuprofen and other similar non-steroidal anti-inflammatory drugs are potentially hazardous and practically they're avoided no matter the subsequent neutral recommendation made by the WHO 
It's known that nitazoxanide might be beneficial to manage COVID-19 with few clinical trials to test its potential with difficulties in recruiting patients

It's known that azithromycin might be beneficial for COVID-19, yet many physicians world-wide prescribe many other antibiotics with unknown potential to manage COVID-19

\section{WHAT THIS STUDY ADDS}

A full protocol to manage early cases of COVID-19 on an individual base

Ibuprofen, and other similar non-steroidal anti-inflammatory drugs are not only superior to paracetamol as analgesics antipyretics but also have a major role in reversing the pathogenesis of COVID-19 as revealed by the case studies presented

NSAIDs/nitazoxanide/azithromycin protocol proved full safety, efficacy and tolerability when used clinically to manage pediatric, adults and pregnant patients

Abstract:

Background and Purpose: The current pandemic of coronavirus disease 2019 has led the world to try a myriad of drugs without being confident of its efficacy and this has led to serious implications and sometimes produced more harm than the disease itself. The author has been trying since March 2020 to publish a paper that disputes this unfortunate recommendation against ibuprofen to be used for COVID-19. On April 2020, the author has published a protocol using nitazoxanide/azithromycin to treat COVID-19 and he finally managed since May 2020 to publish two manuscripts proving the theoretical basis to use non-steroidal anti-inflammatory drugs including ibuprofen as a potential cure when added to nitazoxanide/azithromycin.

Experimental approach: 17 consented COVID-19 Egyptian patients, confirmed by PCR or rapid Ig M test or suspected by history, leucopenia and lymphocytopenia; 7 males, 5 females, 2 pregnant patients one in her $9^{\text {th }}$ week of gestation and the other in her $18^{\text {th }}$ week as well as 2 female children, 6 years and eight monthsand 1.5-years old daughters as well as a 4 years old boy have received ibuprofen/nitazoxanide/azithromycin either in full or in part.

Key Results: The patients have been mostly symptoms-free in five days; the lymphocytic count has been significantly improved and no significant adverse effects have been reported.

Conclusions \& Implications: The manuscript presents a roadmap to illustrate how to deal efficiently with early cases of COVID-19 according to the author's clinical experience using relatively cheap FDA approved drugs.

Keywords: COVID-19, SARS CoV-2, NSAIDs, Ibuprofen, Diclofenac potassium, Nitazoxanide, Azithromycin.

The author has published two manuscripts providing the scientific pathophysiological and pharmacological concept to use the cheap, safe and FDA approved combination nitazoxanide/azithromycin ${ }^{1}$ as well as nonsteroidal anti-inflammatory drugs (NSAIDs) like ibuprofen ${ }^{2}$ for early management of the first wave of corona virus disease 2019 (COVID-19). Keeping in his mind, even before proved, that a vaccine might not be the proper solution to compete with the high morbidity and mortality caused by this highly evolving Severe acute respiratory syndrome coronavirus 2 (SARS $\mathrm{CoV}-2)^{3}$, the author has searched for cheap and safe drugs that might help, thus the first paper was conceived. For the second one, the author has suggested a novel lymphocyte distraction hypothesis to explain the pathogenesis of COVID-19 and later, after it's been preprinted, it also explained the reports of COVID-19 multisystem hyper-inflammatory conditions ${ }^{4,5}$. Further, it's provided a scientific explanation for the reports showing hydroxychloroquine to be ineffective for COVID-19. Noteworthy, after more than one month of the initial warning of the author, the world health organization (WHO) has paused a large trial of hydroxychloroquine due to safety concerns and some countries have started to ban its further use for COVID-196. Most importantly in his second preprinted paper, the author has recommended NSAIDs like ibuprofen for COVID-19 to be added to his first early management 
protocol. This suggestion is contradicting all the current practical approach adopted world-wide that avoided NSAIDs and preferred paracetamol. Noteworthy, the author has fully explained the incident that led to the world to avoid use of ibuprofen ${ }^{7}$ and he's also recommended against the routine use of steroids in COVID-19 but suggested to be administered for patients with late acute respiratory syndrome ${ }^{2}$ and most recently this suggestion has been proved clinically valid ${ }^{8}$

Egypt is considered as one of the most heavily affected African countries as regards to the incidence and mortality rates of COVID-19 and the author, currently working in KSA, has publicly and severely criticized the Egyptian ministry of health (MOH) adopted COVID-19 protocol using hydroxychloroquine/oseltamivir/paracetamol to manage COVID-19 patients considering it as a direct cause for the relatively higher mortality rate encountered in Egypt and still not officially modified even after the WHO recent suspension of any new hydroxychloroquine clinical trials for COVID- $19^{6}$. Through his Facebook account and What's app, he's received, the number is increasing exponentially, numerous compassionate requests, to help Egyptian patients including one nurse who has received verbal, COVID-19 many patients in Egypt can't obtain formal reports, MOH confirmation of a positive SARS CoV-2 (COVID-19) PCR test and has been advised to be treated by the routine protocol at home. Another request came from a pregnant dentist has been diagnosed with SARS CoV-2 IgM rapid test, the others are mostly close contacts to quarantined patients who started to show symptoms and signs of COVID-19 while at home to be noted that currently the quarantine hospitals are almost full in Egypt and there's a huge difficulty to find a vacant bed even for a recently deceased young physician. Nitazoxanide is not commercially available in KSA pharmacies, however the author has obtained two requests to help Egyptian expat patients quarantined in KSA after being tested positive and the author has obtained a formal SARS CoV-2 positive test of a combined nasopharyngeal and oropharyngeal swab obtained from one of them; a hypertensive and type 2 diabetic Egyptian patient who has managed upon advice to receive diclofenac potassium. This patient has already received amoxicillin/clavulanate potassium and thus, the author decided not to administer any further antibiotics as no fever was detected. His troublesome fortnight dyspnea and dry cough have been started to dramatically improve from the first day diclofenac potassium $50 \mathrm{mg}$, postprandial b.i.d., was administered and became mostly symptoms-free in three days, but he continued further two days and later he was discharged. For the second expat, still quarantined on the $8^{\text {th }}$ of June, the author prescribed azithromycin/diclofenac potassium to manage his persistent 20 days fever and was advised, as well as all his COVID-19 patients, not to take antitussives for mild to moderate cough, the author hasn't encountered cases with severe cough, and he advised them to only use warm tea or coffee, ... etc. to soothe any throat and this approach has proved beneficial in all cases, it's been a clinical sense that has trusted the natural reflexes in our body and wasn't based on a scientific evidence based evidence, which might not be available at least shortly. Noteworthy, the second expat patient has explained that his persistent diarrhea has been started to improve immediately after ceasing, upon advice, to use the previously prescribed antitussive dextromethorphan and before managing to obtain the other newly prescribed drugs from outside the quarantine facility. Further, this second expat has informed that he's also ibuprofen $600 \mathrm{mg}$ and he asked if he might take it instead of diclofenac potassium and he was authorized, and he's reported better clinical experience than diclofenac potassium as regards to headache, fatigue as well as pain control. The clinical picture as well as the investigations differed from a patient to another and the author used an individualized medicine approach for each case, e.g. most patients showed lymphopenias while some has showed "apparently" normal complete blood count. The nurse with the verbal confirmation of positive SARS CoV-2 PCR had on May $15^{\text {th }}, 2020$ a prior treatment total white blood cells count of $7.8 \times 10^{3} / \mu$ l, relative lymphocyte count $5.5 \%$, mixed population of monocytes, basophiles and eosinophils count $16.5 \%$ and neutrophils count $78 \%$ (Figure 1), the swab revealed positive on the $18^{\text {th }}$ of May and he started to take the full mentioned protocol on the $19^{\text {th }}$ of May and after the 5 days treatment regimen, the total white blood cells count was $6.3 \times 10^{3} / \mu$, lymphocyte count $43 \%$, mixed population of monocytes, basophiles and eosinophils count $8 \%$ and neutrophils $49 \%$ (Figure 2) and this highly significant elevation of lymphocytes might be considered as the first clinical proof of the COVID-19 pathogenesis theory represented in the second paper published by the author ${ }^{2}$, the significant decrease of neutrophils may represent the efficacy of azithromycin as well as the restoration of the interferon immune system homeostasis to eliminate any associated secondary bacterial infection, to be followed by other in- 
vestigations in randomized clinical trials as described in the published two papers ${ }^{1,2}$. The patient has also authorized the release of his results for medical purposes.

In summary, 17, confirmed or suspected COVID-19 patients; 7 males, 5 females, 2 pregnant patients one in her $9^{\text {th }}$ week of gestation and the other in her $18^{\text {th }}$ week as well as 2 female children, 6 years and eight monthsand 1.5-years old daughters of a colleague and a 4 years old son of one of the authors' relatives. A consent has been obtained from each case/guardian and the author prescribed the adjusted dose of nitazoxanide suspension, azithromycin suspension and ibuprofen syrup for children. For adults he used NSAIDs, either postprandial ibuprofen $400 \mathrm{mg}$ b.i.d. (600 $\mathrm{mg}$ in KSA) or diclofenac potassium $50 \mathrm{mg}$ b.i.d. alone for early cases complaining of sore throat, dry cough or mild dyspnea with no fever or fever less than $38^{\circ} \mathrm{C}$. However, the author recommends adding nitazoxanide to NSAIDs for patients complaining from diarrhea even if their temperature wasn't above $38^{\circ} \mathrm{C}$ and it has also proved effective to control a moderate diarrhea encountered in an adult patient though it was administered in the full regimen. The author has prescribed the whole NSAIDs (as described)/nitazoxanide (500 mg bid)/azithromycin (500 mg once daily) protocol for COVID-19 patients (proved by PCR or suggested by the clinical picture, history of close contact to a quarantined patient and/or lymphopenia) complaining of fever more than $38^{\circ} \mathrm{C}$. Most importantly, NSAIDs have shown remarkable superior clinical efficacy as compared to paracetamol, for those who used it before switching to the new protocol, for controlling high fever, headache and malaise. The elder child complained of severe sore throat that led her to severe anorexia and she also complained of diarrhea and vomiting. She and her sister totally recovered from all symptoms in three days but continued the five days regimen. Some patients have also complained of severe malaise, anorexia, moderate diarrhea, anosmia, dysgeusia, ageusia, moderate to severe flank and back pain which have been totally improved in less than 10 days and most have improved during the five days regimen. The author has allowed the patients to receive vitamin $\mathrm{C}$ and Zinc and any other food supplements though their proven clinical benefit is to be discovered later, if meant to be discovered as the author suggests they're almost harmless and should be continued as usual.

As a physician as well as a pharmacologist, the author has assessed any possible contraindication or drug-drug interactions for those patients complaining from type 2 diabetes, hypertension as well as rheumatoid arthritis and for the latest who was also the pregnant dentist complaining of fever, sore throat, fatigue, dry cough, the author has used azithromycin/nitazoxanide and stopped hydroxychloroquine as well as sulfasalazine for the five days treatment course to prevent adverse drug interactions. Similarly, NSAIDs were not prescribed for her as she was already on prednisone for her rheumatoid arthritis. However, NSAIDs might be considered for other pregnant cases before the third trimester, most recently the author has prescribed them for another pregnant suspected patient in her $18^{\text {th }}$ week of gestation with no harm and she's completed one week diclofenac potassium $50 \mathrm{mg}$ b.i.d. course though I've strongly recommended her to stick to the five days and she's been totally relieved of symptoms with no significant adverse effects reported, as the author believes the benefits are exceeding the potential risks for this short-term regimen and the author has followed and follows each new case eagerly. The author wishes to declare this full regimen including NSAIDs has shown full tolerability, no reported adverse effects and seven patients have fully, not mostly, recovered in less than one week, one has been free of all symptoms in only three days and the author was waiting for the formal negative SARS CoV-2 (COVID-19) qualitative PCR test confirmation from the Egyptian Expat living in KSA but because of the huge numbers of new infections, it was decided not to repeat the test after the clinical improvement and the author agrees with this pharmacoeconomically vigilant attitude. Interestingly, The author has already recommended all his patients to remain isolated for at least 21 days from the beginning of the symptoms including at least one week of totally symptoms free period even if the PCR test revealed negative earlier and this recommendation was to avoid the possibility of any false negative results and he's also recommended them to avoid any direct/intimate contact with others till at least one month has passed from the beginning of symptoms and at least two weeks of totally symptoms free period, this advice was based on a positive test from the nurse though he was symptoms free for more than 10 days to be fully elucidated by other research work to determine the best possible recommendation. The personal doctor patient communication and consultation were performed through Facebook Messenger and What's app applications and the author believes this might be considered as a COVID-19 personalized telemedicine 
approach and through these applications, all the findings were documented in Arabic as it's our mother tongue.

Very interestingly, a 48 years old female, not counted in this manuscript, patient has complained of severe sore throat, malaise, fever and mild cough to which a family physician has asked for a CT chest which has revealed patchy ground glass opacities scattered at right lung mainly peripheral in location associated with minimal thickening of the lung interstitium as well as peri-bronchial cuffing (Figure 3) as described in the given report and her family physician diagnosed her as a COVID-19 patient and has prescribed paracetamol, betamethasone injection IM, doxycycline $100 \mathrm{mg}$ for 15 days, azithromycin $500 \mathrm{mg}$ orally, some vitamins and minerals as well as a syrup that contains antihistaminic and expectorant for three days. Her condition has deteriorated with persistent fever, she's started to experience yellow vision, tachycardia and the author has received a compassionate request to help, asked for an urgent CBC (Figure 4) that revealed a total leucocytic count of $5.1 \times 10^{3} / \mu$ l with a low lymphocytic count of $12 \%$. I've immediately stopped the bacteriostatic doxycycline, ordered an immediate ketoprofen IM injection to be followed by diclofenac potassium b.i.d. azithromycin $500 \mathrm{mg}$ once for five days in combination with parenteral cefoperazone, chosen for its relatively longer half-life as well as its efficacy against atypical respiratory micro-organisms, $1 \mathrm{gm}$ once daily for the first three days, nitazoxanide $500 \mathrm{mg}$ b.i.d. that has also managed to control diarrhea that has appeared after the start of treatment and the author has considered it as a good sign. The author has also stopped the antitussive syrup. Her condition has improved dramatically within five days and the CBC on the fifth day (figure 5) has revealed a total leucocytic count of $6 \times 10^{3} / \mu$ with an elevated lymphocytic count of $23 \%$. Noteworthy, she's also been diagnosed positive for SARS CoV-2 IgM upon the author's request.

Unfortunately, a 59 years old patient, not counted in this manuscript, has been introduced late in his COVID-19 natural history with a positive PCR result and had a CT chest showing ground glass opacities and suffering from hypertension and pre-diabetes. His daughter was a physician who unfortunately decided to give him only nitazoxanide and not to listen to a recommendation to stop oseltamivir or to administer NSAIDs and the author decided not to continue further. Later, the patient condition has deteriorated but fortunately was saved after being urgently transferred to one of the quarantine hospitals, he was fortunate not to die like other younger and healthier patients quarantined in Egyptian hospitals and one might wonder if his relatively good fortune might be related to nitazoxanide.

Noteworthy, the author, 39-years-old healthy male, would also like to declare that on the $22^{\text {nd }}$ of May on 6 a.m., he felt feverish, suffered from dry cough and nasal congestion, he has only found diclofenac potassium $50 \mathrm{mg}$ available, he's taken it once and all the symptoms disappeared in less than one hour and on 6 p.m. of the same day, he's swallowed another tablet though complaining of no symptoms. The author believes it wasn't probably SARS CoV-2 but he can't be fully sure as he was in direct contact with many people and many patients are even symptomless, he didn't swallow any other pills and remained free of symptoms, he truly wished to use the part of his suggested protocol which he's fought against the world, like his great Coptic father Athanasius Contra Mundum, to prove it on himself as proved on his patients and he's not counted in this manuscript. Further, a pulmonologist; Dr. Pablo Juárez from Guadalajara, Mexico who have read the first published article, has recently sent an email of gratitude from which the author wishes to quote its end: "At this moment I have 24 patients, only with nitazoxanide, and other treatments at the hospital and I have the real idea that it is working, and nitazoxanide can help us to reach our goal of less patients in hospital, less contacts, and less infections, I am advising my colleagues to use nitazoxanide in the suspects so they don't have to wait 3-5 days for the covid-19 test and then hospitalized" and the author wishes to agree with him and he repeats his previous calls to begin the clinical trials soonest as we might finally have found a battle changer in this horrible battle.

\section{Acknowledgment}

The author would like to thank all the patients, Muslims and Christians, who believed that something good might come out of Egypt (Joh 1: 46). Noteworthy, all the consultations were, and are, provided for free, I'm just following my Lord's command: "Freely you have received, freely give" (Mat 10: 8). The author would like also to thank the British Journal of Clinical Pharmacology that has, since the $6^{\text {th }}$ of May, shared the 
three Egyptian manuscripts, including the main bulk of this manuscript, showing the efficacy of ibuprofen in COVID-19 with the WHO and preprinted two of them on Authorea; Wiley's new initiative to streamline the early sharing of research and open up the peer review process.

Ethical approval:

The author is a member of the Egyptian syndicate of physicians since 2006, he's followed every ethical command in any and all procedures mentioned in this manuscript.

Consent

Compassionate requests/consents were received from any patient/legal guardian involved in the study through Facebook messenger and What's app.

Data availability statement:

The author declares full transparency and is more than willing to let any official authority to directly contact any or all patients mentioned in the manuscript whenever required. Any further related data that might be need, the author would be at your disposal.

Conflict of interests:

The author has no conflicts of interest to declare.

Funding:

None.

References:

1. Kelleni MT. Nitazoxanide/Azithromycin combination for COVID-19: A suggested new protocol for COVID-19 early management.Pharmacological Research 2020: 104874.

2. Kelleni M. Ibuprofen potential addition to COVID-19 early management protocols: could it be superior to paracetamol and hydroxychloroquine? 2020, May 6. https://journal.sketchingscience.org/users/318758/articles/448656-ibuprofen-potential-addition-to-covid19-early-management-protocols-could-it-be-superior-to-paracetamol-and-hydroxychloroquine.

3. Bloomberg Law (John Lauerman and James Paton). The First Covid Vaccines May Not Prevent Covid Infection (1). 2020, June 15. https://news.bloomberglaw.com/coronavirus/the-first-covid-vaccines-may-notprevent-you-from-getting-covid?context $=$ search\&index $=15 \&$ utm_source $=$ twitter\&campaign=B1BDE4E0AF5C-11EA-A029-9CF94F017A06\&utm_medium=lawdesk\&fbclid=IwAR3HM9yKf4JxM99n0ZRqCdQqFSn-WOcTc8T1NvOGnN1f-WaD3Ip0DCmBks.

4. WHO. Multisystem inflammatory syndrome in children and adolescents temporally related to COVID-19. 2020, May 15. https://www.who.int/news-room/commentaries/detail/multisystem-inflammatory-syndromein-children-and-adolescents-with-covid-19.

5. Simpson JM, Newburger JW. Multi-System Inflammatory Syndrome in Children in Association with COVID-19. Circulation 2020.

6. Reuters (Matthias Blamont). France, Italy, Belgium act to stop use of hydroxychloroquine for COVID-19 on safety fears. 2020, May 27. https://www.reuters.com/article/us-health-coronavirushydroxychloroquine/france-italy-belgium-act-to-stop-use-of-hydroxychloroquine-for-covid-19-on-safetyfears-idUSKBN233197.

7. Kelleni M. ACEIs, ARBs, Ibuprofen linked to COVID-19: The other side of the broken mirror. 2020, June 2,. https://www.authorea.com/users/318758/articles/456017-aceis-arbs-ibuprofen-linked-to-covid-19-theother-side-of-the-broken-mirror?commit=c85d790e9c1c2a3e698675ed1798efe534abdfa7 . 
8. BBC (Michelle Roberts). Coronavirus: Dexamethasone proves first life-saving drug. 2020, June 16. https://www.bbc.com/news/health-53061281.

Figure 1. pre-treament CBC

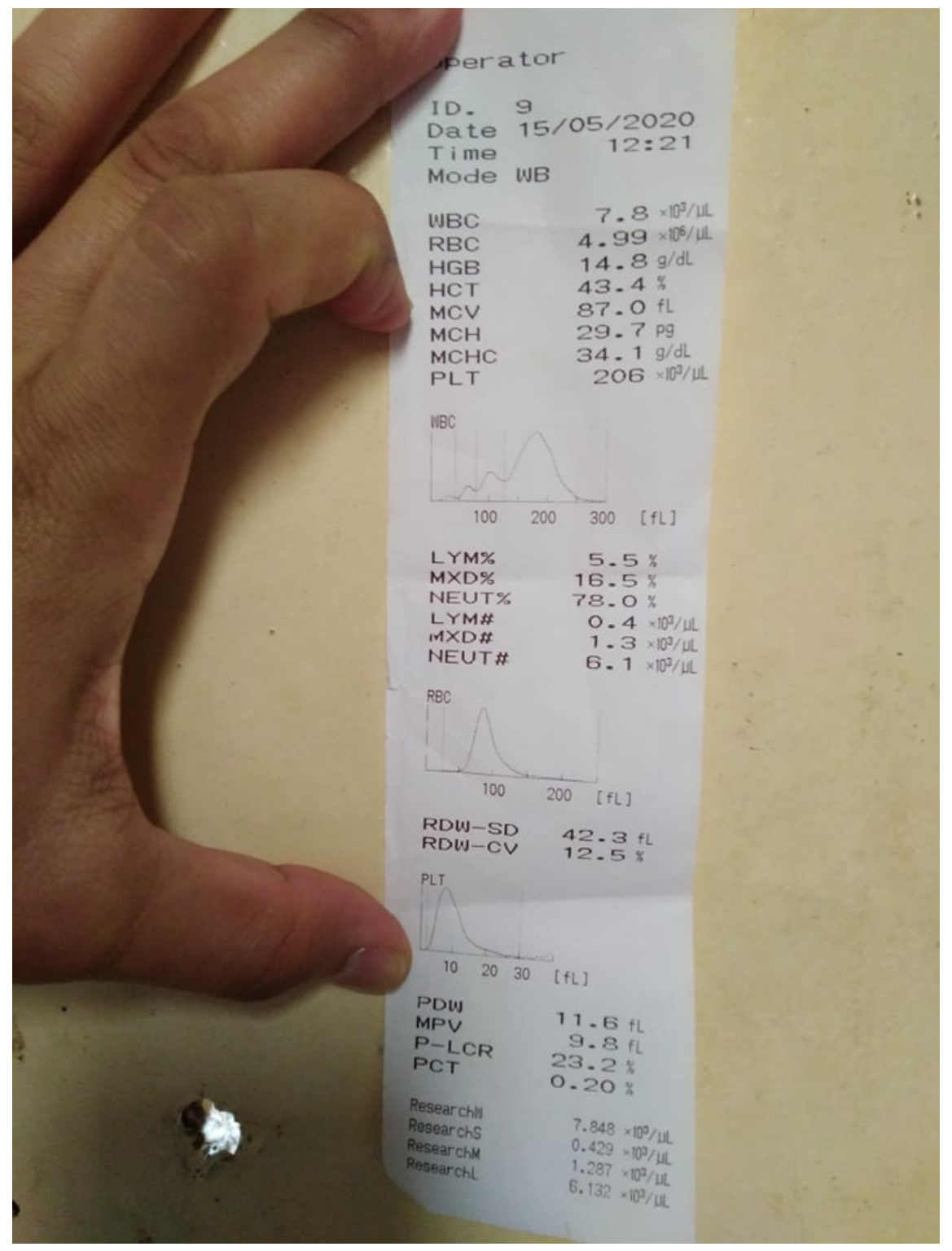


Complete blood picture

\begin{tabular}{|c|c|c|c|c|c|}
\hline Test & & Result & & Unit & Reference range \\
\hline \multicolumn{6}{|l|}{ Hb\&indeses } \\
\hline Haemoglobin & & 15.6 & & $\mathrm{gm} / \mathrm{dl}$ & $12.5-17.0$ \\
\hline Haematocrit (PCV) & & 44.7 & & $\%$ & $39-49$ \\
\hline Red Cell Count & & 5.18 & & $\mathrm{mil} / \mathrm{cmm}$ & $4.3-5.7$ \\
\hline $\mathrm{MCV}$ & & 86 & & $f l$ & $80-99$ \\
\hline $\mathrm{MCH}$ & & 30 & & $p g$ & $27-34$ \\
\hline $\mathrm{MCHC}$ & & 35 & & $\%$ & $32-37$ \\
\hline \multicolumn{6}{|l|}{ TLC \& Differential } \\
\hline White cell count & $\frac{\text { Result }}{6.3}$ & $\underline{\text { Relative }}$ & Absolut & $\frac{\text { te Result }}{\text { Thousand/cmm }}$ & $\frac{\text { Absolute Normal }}{4.11}$ \\
\hline Basophils & 0 & 0.2 & 0 & $/ \mathrm{cmm}$ & $0-100$ \\
\hline Eosinophils & 2 & $1 \cdot 4$ & 126 & $/ \mathrm{cmm}$ & $0-800$ \\
\hline Staff & 1 & $0-6$ & 63 & $/ \mathrm{cmm}$ & $0-200$ \\
\hline Segmented & 48 & $37-75$ & 3024 & $/ \mathrm{cmm}$ & $2000-7200$ \\
\hline Lymphocytes & 43 & $20-45$ & 2709 & $/ \mathrm{cmm}$ & $1500-3500$ \\
\hline Monocytes & 6 & $2-10$ & 378 & $/ \mathrm{cmm}$ & $200-900$ \\
\hline \multicolumn{6}{|l|}{ PLT } \\
\hline Platelet count & & 270 & & Thousand/cmm & $150-450$ \\
\hline MPV & & 8.6 & & $f l$ & $6.5-12$ \\
\hline PDW & & 11.3 & & & $9-17$ \\
\hline P-LCC & & 49 & & $x 10^{\wedge} 9 / \mathrm{L}$ & $30-90$ \\
\hline Comments & & & & & \\
\hline
\end{tabular}









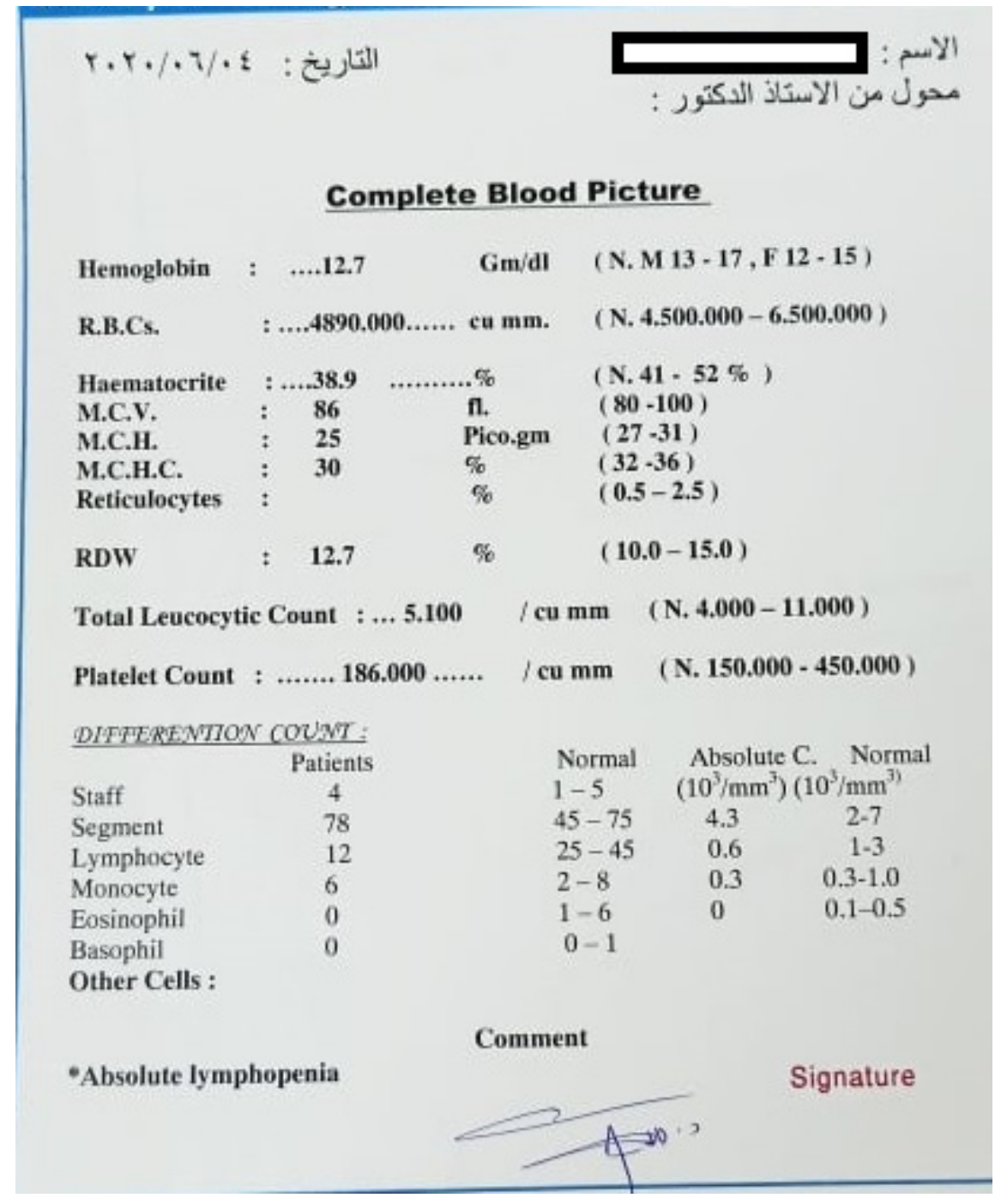


التاريخ : :

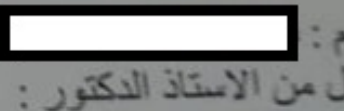

\section{Complete Blood Picture}

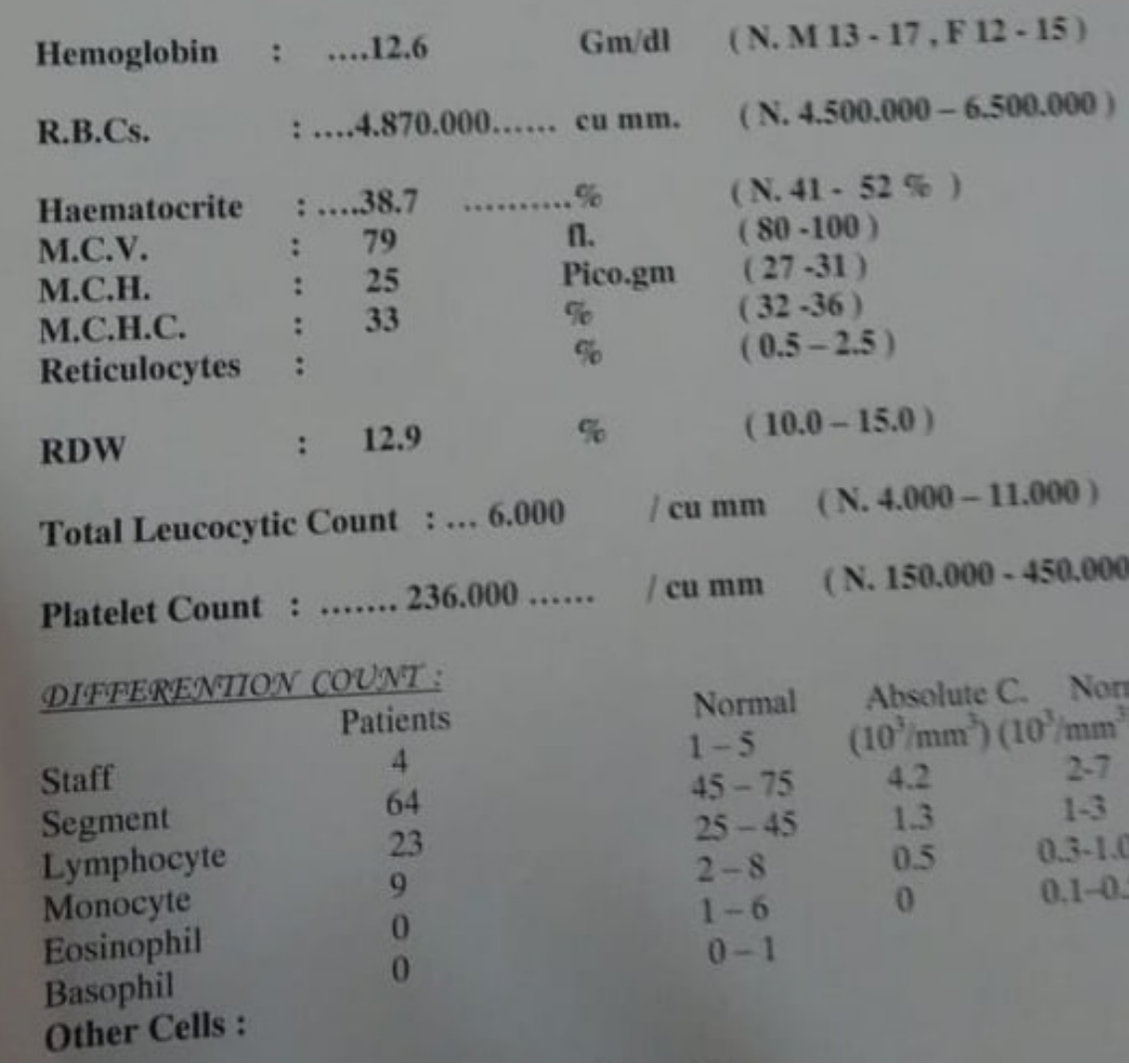




$$
\therefore-
$$

